\title{
ÖKOLOGIE
}

\section{Soziale Konzepte für die Zeit nach dem Öl}

VON ULRICH DELLER UND

KLAUS DOSCH

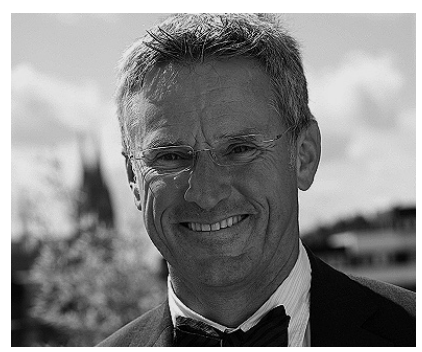

Prof. Dr. Ulrich Deller ist Hochschullehrer für Soziale Arbeit an der Katholischen Hochschule Nordrhein-Westfalen in Aachen. Er hat längjährige Erfahrung in der Leitung sozialer Dienste und als Leiter des berufsbegleitenden Studiengangs Kooperationsmanagement.

Internet http://www.kooperationsmanagement-aachen.de

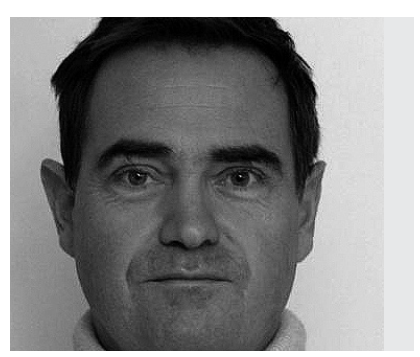

Klaus Dosch ist als Diplom-Wirtschaftsingenieur und DiplomGeologe Wissenschaftlicher Leiter der Aachener Stiftung Kathy Beys. Er arbeitet seit zwei Jahrzehnten an strategischen Konzepten an der Schnittstelle von Ökonomie und Umwelt. Internet http://www.aachenerstiftung.de

\author{
In zahlreichen Ländern hat eine Debatte darüber \\ begonnen, wie sich die Begrenztheit der fossilen Brenn- \\ stoffe auf das gesellschaftliche Leben auswirken kann. \\ Die Energiewende wird auch Auswirkungen auf die \\ Soziale Arbeit und die Sozialwirtschaft haben.
}

Erdöl ist der wichtigste Energieträger industrialisierter Volkswirtschaften. Es stellt den Grundstoff dar, aus dem Treibstoffe, Heizenergie, Baustoffe, Dünger und andere chemische Erzeugnisse gewonnen werden. Deutschland importierte im Jahr 2009 rund 70 Prozent seiner benötigten Energie aus dem Ausland. Mit circa 33 Prozent am Primärenergieverbrauch Deutschlands hat das Erdöl eine herausragende Stellung vor Erdgas (18\%), Kernenergie $(10 \%)$ und importierter Steinkohle $(9 \%)$. (1)

Seit Beginn der 1960er Jahre nimmt die Menge des jährlich neu gefundenen Öls kontinuierlich ab, gleichzeitig überschreitet die jährliche Fördermenge die Neufunde um etwa das Dreifache. Öl kann nur rentabel gefördert werden, wenn für das Öl ein höherer Preis erzielt werden kann, als Exploration und Förderung kosten. Mit diesem Zusammenhang wird häufig begründet, dass Öl gewissermaßen unbegrenzt zur Verfügung steht, da bei steigendem Ölpreis immer neue Vorkommen rentabel werden. Diese ausschließlich pekuniäre Sicht ist jedoch unvollständig. Öl kann auch nur gefördert werden, wenn die Summe der für Exploration, Bohrung und Förderung investierten Energiemenge kleiner ist, als die Energie, die schließlich aus einem Bohrloch zu gewinnen ist. Muss mehr Energie investiert werden, als anschließend gewonnen werden kann, mag der Ölpreis noch so hoch steigen, wäre eine Ölförderung unter solchen Be- dingungen wirtschaftlicher und energetischer Unsinn.

Die Bundesanstalt für Geowissenschaften und Rohstoffe rechnet zwischen 2010 und 2020 mit dem Eintreten des Fördermaximums bei Öl. Spätestens ab diesem Zeitpunkt werde das Preisniveau deutlich ansteigen. Der Abbau alternativer Ölvorkommen wie Schieferöl und Teersande werden zwar dann rentabel, deren Förderung kann aber den Rückgang der Förderung konventionellen Öls nicht kompensieren. (2) Andere Forscher vermuten den Zeitpunkt schon früher, wie beispielsweise EWG (2008). (3)

\section{Konsequenzen für die Gesellschaft}

Aus diesen Entwicklungen lassen sich unterschiedliche Szenarien ableiten. Die Entwicklungen sind in hohem Maße interdependent und nicht national begrenzbar. In allen Bereichen werden sich veränderte Preisentwicklungen ergeben, die sich zudem gegenseitig beeinflussen werden. Besondere Bedeutung hat diese Entwicklung dann, wenn sie sich auf die Investitionsgüter auswirkt.

In einer Studie für die Enquetekommission des Landtages von NordrheinWestfalen haben Meyer et. al. eine Modellierung der Folgen drastisch gestiegener Ölpreise auf die Gesellschaft durchgeführt. (4) Die Szenarien basieren auf breit erfassten volkswirtschaftlichen Daten und gehen von einem anerkannten Modell (»PANTA RHEI «) gesamt- 
wirtschaftlicher Berechnung aus. In allen als möglich berechneten Szenarien kommen Meyer u. a. zu dem Schluss, dass die Preissteigerungen bei fossiler Energie auf Unternehmen sowie Verbraucherinnen und Verbrauchern unterschiedlich wirken. Da alle Unternehmen weltweit gleichermaßen betroffen sind, können Unternehmen die höheren Energiekosten an die Endverbraucher weitergeben. Diese können ihre Energiekostensteigerungen nur wenig durch Minderverbrauch kompensieren, zugleich sehen sie sich dem grundsätzlich gestiegenen Preisniveau gegenübergestellt. leichtes Heizöl bis 2030 belastet vor allem die Mieter unterer Einkommen deutlich. Dies umso mehr, als die Vermieter die Kosten für energetische Erneuerung auf die Mieter umlegen können.

Zugleich können unkalkulierbare Folgekosten entstehen. Denn dichtere Fenstersysteme erfordern ein sehr diszipliniertes Lüftungsverhalten der Mieter, wenn auf den Einbau entsprechender Lüftungssysteme verzichtet wird. Die Folge ist oft Schimmelpilzbefall der Wohnungen, welcher erneute Kosten für Reparaturen und vor allem für die Abwehr der gesundheitlichen Beeinträchti-

\section{"Soziale Arbeit sollte neue gemeinschaftliche Formen der Lebensbewältigung anregen"}

\section{Konsequenzen für die Soziale Arbeit}

Diese Entwicklung wird Auswirkungen weit über die reinen Energiekosten als solche haben. Für die Soziale Arbeit wird sich der Druck zur Ökonomisierung verstärken, und auch ihre zugrundeliegenden Konzepte werden betroffen sein. » Ressourcenorientierte Arbeit « gewinnt vor diesem Hintergrund eine erweiterte Bedeutung. Der Druck steigt, dass die Hilfebedürftigen zuerst einmal ihre Selbsthilfepotentiale einsetzen sollen, bevor sie Transferleistungen in Anspruch nehmen. Die ökonomische Entwicklung nach dem Öl-Höhepunkt kann in diesem Sinne auch Auswirkungen auf die gerade veränderten SGB-Gesetze zur Grundsicherung des Lebensunterhalts haben. Neben negativen Entwicklungen wäre es auch durch Belebung traditioneller Konzepte in der Sozialen Arbeit möglich, eine neue Qualität in die Lebensbewältigungsstrategien armer Familien zu bringen.

\section{Beispiel Wohnen und Heizen}

Aktuell sind die Preise für leichtes Heizöl fast wieder auf dem Niveau von 2008. Schon jetzt sagen fast 7 Prozent aller Haushalte, dass sie nicht in der Lage sind, ihre Wohnung angemessen zu heizen. Die von Meyer (2006) prognostizierte Verdoppelung der Kosten für gungen der Bewohner nach sich zieht. Legt man den deutschen Betriebskostenspiegel (5) zugrunde, dann sind im Jahre 2009 monatlich alleine für Bezieher von Hartz IV rund 171 Millionen Euro an Heizkosten angefallen. Die Bundesagentur für Arbeit gibt in ihrem Analysebericht für Oktober 2010 denn auch 162 Millionen Euro als monatliche anerkennungsfähige Heizkosten der Bezieher von Grundsicherung für Arbeitssuchende an. Die Verdoppelung der Heizölpreise würde die öffentlichen Haushalte dann um rund 1,9 Milliarden Euro je Jahr aufblähen. Selbst wenn man eine Erhöhung der Kaufkraft und eine Verlagerung hin zu regenerativen Energien einkalkuliert, werden die Kosten deutlich steigen. Schon jetzt sind in manchen kommunalen Mietverhältnissen die Kosten für die Nebenkosten höher als die reinen Mietkosten.

Für die Soziale Arbeit könnte das heißen, auf kurzfristige Investitionen zu verzichten und langfristig in den sozialen Wohnungsbau und zwar in Passivbauweise zu investieren, um in der Lage zu sein, hilfebedürftigen Menschen entsprechenden Wohnraum zur Verfügung stellen zu können. Sie könnten wie andere auch die Förderprogramme der KFW in Anspruch nehmen. Es könnte heißen, dass Empfänger von Transferleistungen ihre Wohnungen nur begrenzt (zeitlich oder räumlich) voll heizen können. Alternativen könnten nötig sein, vielleicht, indem vermehrt geheizte Treffpunkte (»Wärmestuben «) bereitgestellt werden. Da die Hälfte aller Bedarfsgemeinschaften aber Single-Haushalte sind, stehen auch gemeinschaftliche Wohnformen zur Diskussion. Hier liegen Möglichkeiten der Sozialen Arbeit, neue gemeinschaftliche Formen der Lebensbewältigung anzustoßen.

\section{Beispiel Nahrung}

»Um in westlichen Industrieländern eine Lebensmittelkalorie zu produzieren, werden zehn Kalorien aus Kohlenwasserstoffen verbraucht ... Das ist so, als ob wir Erdöl essen würden «, formuliert David Strahan zugespitzt. (6) Die Nahrungsmittelproduktion hängt stark von fossilen Brennstoffen ab. Viele unterschiedliche Aspekte zusammen haben 2008 einen enormen Anstieg der Preise für Agrar-Rohstoffe bewirkt: die höheren Produktionskosten ebenso wie das wegen der Biokraftstoffe verringerte Angebot an Lebensmitteln. In Deutschland haben sich die Nahrungsmittelpreise trotz des erbitterten BilliganbieterKampfes seit 2005 um 12 Prozent erhöht. Für die Hartz IV-Empfänger haben diese Zusammenhänge enorme Auswirkungen. 37 Prozent ihres Budgets stehen für Lebensmittel zur Verfügung und sind im Prinzip sehr knapp berechnet.

Aber auch diese Entwicklung könnte positive Auswirkungen haben, beispielsweise durch Arbeit in mehr regionalen (Nahrungsmittel-) Produktionen. Die Verantwortung für das nahe Umfeld kann also in größerer Weise gelebt werden. In diesem Zusammenhang kann Sozialraum-Orientierung neu buchstabiert werden. Für die Soziale Arbeit könnte die Wiederbelebung der SchrebergartenKultur interessant sein. Vielleicht lohnt sich die Investition in Land, das für Kleingärten zur Verfügung gestellt werden könnte, um in die Subsistenz armer Familien investiert werden zu können: gesunde und selbst erwirtschaftete preiswerte Nahrungsmittel.

\section{Beispiel Mobilität}

Steigende Preise für Öl wirken sich direkt auf die Kosten für Mobilität aus. Wegen des vergleichsweise hohen Steueranteils an den Treibstoffpreisen in Deutschland führt eine Steigerung des 
Rohölpreises nicht in gleicher Höhe zur Verteuerung der Treibstoffe. Auch im öffentlichen Nahverkehr kennt die Preisentwicklung nur eine Richtung: Im Zeitraum von Januar 2003 bis Dezember 2010 stiegen die Verkaufspreise für Einzelfahrscheine um 38 Prozent (Destatis 2011). Gegenwärtig wenden deutsche Haushalte mit 328 Euro pro Monat durchschnittlich 14,6 Prozent ihrer Konsumausgaben für Mobilität aus, darunter 98 Euro (30\%) für Treib- und Schmierstoffe (Destatis 2010).

Insbesondere bei Geringverdienern, die auf Mobilität angewiesen sind, um ihren Arbeitsplatz zu erreichen, führen steigende Treibstoffkosten zu finanziellen Engpässen. Zur Kompensation steigender Preise scheinen hier die Möglichkeiten von Fahrgemeinschaften längst nicht ausgeschöpft. Hier lassen sich basierend auf den Möglichkeiten von Web 2.0 und Smartphones interessante Dienstleistungskonzepte denken, um die Nachfrage und das Angebot gemeinschaftlichen Fahrens zusammen zu bringen, die von sozialökonomischen Projekten aufgegriffen werden könnten. Die Grundsicherung nach Hartz IV lässt schon heute wenig Mobilität zu. Lediglich in Verkehrsverbünden, die ein stark subventioniertes Sozialticket anbieten, kann diese Personengruppe innerhalb des öffentlichen Personennahverkehrs an grundlegender Mobilität teilhaben. Auf dem Land ist dies häufig nicht der Fall. Gerade in diesen ländlichen Bereichen kommen mit weiter steigenden Treibstoffkosten auf die Träger Sozialer Arbeit Herausforderungen zu, einerseits unkonventionelle Hilfen zur Deckung dieses Mobilitätsbedarfes anzubieten, andererseits (neue) Konzepte für mobile soziale Dienstleistungen zu entwickeln.

\section{Beispiel Finanzen}

Wenn wir für die Diskussion ein Szenario der erwähnten Meyer-Studie zugrunde legen (» $\mathrm{A} 2$ «), bedeutet dies, einen dauerhaften Rohölpreis von 130 Dollar je Barrel bis 2030 als Bezugspunkt zu haben. Für dieses Szenario berechneten Meyer u. a. »eine Minderung der Einkommen der Haushalte, wodurch dann auch das Steueraufkommen des Staates leidet. Da keine spezifische fiskalpolitische Reaktion des Staates unterstellt wurde, bedeutet dies, dass der Konsum des Staates reduziert wird «.
Meyer et.al. gehen davon aus, dass das Bruttoinlandsprodukt sich um 3,3 Prozent absenken wird bei gleichzeitiger Zunahme der Arbeitslosenzahlen.

Die Soziale Arbeit wäre von diesen Berechnungen in zweifacher Weise betroffen. Zum einen gehen Meyer et. al. davon aus, dass die Beschäftigung im Bereich der sozialen Sicherung um 14,5 Prozent abnehmen wird. Zum anderen wird die Finanzkraft der öffentlichen Haushalte (in Nordrhein-Westfalen) um 6,9 Prozent zurückgehen.

\section{Gemeinwesenarbeit als eine Lösung}

Das Post-Carbon-Institute in Kalifornien hat vor dem Hintergrund der Nahrungsmittel-Produktion einen Plan entwickelt, wer was tun könnte. Für Projekte der Gemeinwesenarbeit machen sie konkrete Vorschläge. (7) Dass eine Kommune sich in solch einem Pro-

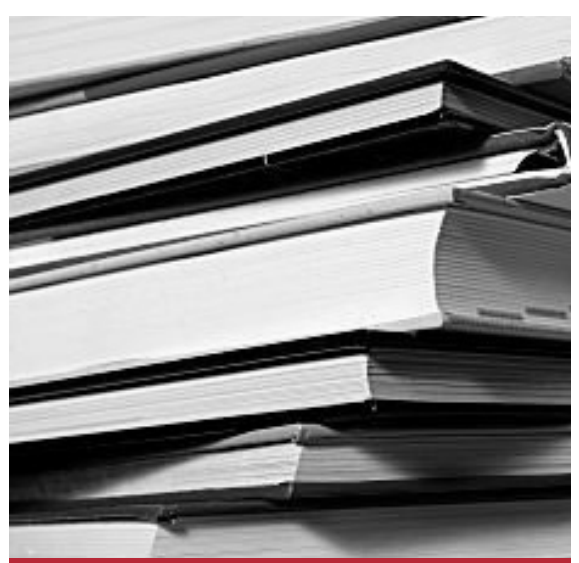

\section{Literatur}

BGR (2010): Bundesanstalt für Geowissenschaften und Rohstoffe: Kurzstudie Reserven, Ressourcen und Verfügbarkeiten von Energierohstoffen. Hannover 2010.

Bloomington (2009): Redifining Prosperity: Energy Descent and Community Resilience.

Report of the Bloomington Peak Oil Task Force. October 2009.

BMWI (2010): Energie in Deutschland - Trends und Hintergründe zur Energieversorgung, Bundesministerium für Wirtschaft und Technologie, Berlin.

Bünder, Peter (2002): Geld oder Liebe. Verheißungen und Täuschungen der Ressourcenorientierung in der Sozialen Arbeit. Münster 2002.

Destatis (2010): Statistisches Jahrbuch 2010. Für die Bundesrepublik Deutschland mit Internationalen Übersichten. Wiesbaden. zess als Ganze auf den Weg macht, zeigt das beeindruckende Beispiel von Bloomington in Indiana. (8)

In dem Moment, in dem die Soziale Arbeit diesen Prozess aktiv zur Kenntnis nimmt, kann sie ein neues Problembewusstsein gewinnen. Soziale Arbeit könnte so als Profession beschreibbar werden, die nicht reaktiv (gleichsam als Feuerwehr) tätig ist, sondern pro-aktiv und unabhängig agiert und gesellschaftliche Entwicklung anstößt und richtungweisend sein könnte.

\section{Anmerkungen}

(1) BMWI (2010).

(2) UBA (2007), S. 39.

(3) EWG (2008) S. 46 ff.

(4) Meyer et al. (2006).

(5) DMB (2010).

(6) Geets/Rossom (2008) S. 40.

(7) Heinberg/Boomford (2009) S. 36 f.

(8) Bloomington (2009).

Destatis (2011): Online Preismonitor des Statistischen Bundesamtes, Abruf Januar 2011. DMB (2010): Deutscher Mieter Bund Betriebskostenspiegel für Deutschland 2009. Berlin 2010. EWG (2008): Energy Watch Group: Zukunft der weltweiten Ölversorgung, von Werner Zittel und Jörg Schindler, Ottobrunn 2008. Geets/Rossom (2008): Der Hunger nach öl. Von Jean-Pierre Geets/Julie Van Rossom. In: research*eumaga

Heinberg/ Boomford (2009): The Food \& Farming Transition Toward a Post Carbon Food System. Von Richard Heinberg und Michael Bomford, by Post Carbon Institute, Sebastopol, California/USA.

Hirsch, Robert L. (2005): Peaking Of World Oil Production: Impacts, Mitigation \& Risk Management, United States Department of Energy, National Energy Technology Laboratory (NETL), Washington DC, USA, 2005.

Meyer et.al. (2006): Auswirkungen verschiedener Preisszenarien auf einschlägige Branchen und Bereiche in NRW. Gutachten im Auftrag der Enquetekommission "Auswirkungen längerfristig stark steigender Preise von Ölund Gasimporten auf die Wirtschaft und die Verbraucherinnen und Verbraucher in NRW “ des Landtags von Nordrhein-Westfalen von Bernd Meyer, Christian Lutz, Martin Distelkamp Osnabrück, 18.12.2006.

UBA (2007): Umweltdaten Deutschland 2007, Umweltbundesamt, Dessau.

Zittel, Werner (2009): Ludwig-Bölkow-

Systemtechnik GmbH, Vortrag Peak Oil - und was nun? Hamburg, 20.11.2009. 